\title{
Local treatment for liver oligometastases in breast cancer patients: identification of prognostic factors and exploration of appropriate treatment strategy
}

\author{
Kuikui Jiang", Wen Xia", Ruoxi Hong", Fei Xu, Qiufan Zheng, Qianyi Lu, Kaping Lee, Yuan Li, \\ Qinglian Zhai, Yanxia Shi, Zhongyu Yuan, Shusen Wang \\ Department of Medical Oncology, Sun Yat-sen University Cancer Center, State Key Laboratory of Oncology in South China, Collaborative \\ Innovation Center for Cancer Medicine, Guangzhou 510060, China \\ Contributions: (I) Conception and design: S Wang, K Jiang, W Xia, R Hong; (II) Administrative support: S Wang, F Xu; (III) Provision of study \\ materials or patients: S Wang, Y Shi, Z Yuan; (IV) Collection and assembly of data: K Jiang, W Xia, R Hong; (V) Data analysis and interpretation: \\ K Jiang, W Xia, R Hong, F Xu, Q Zheng, Q Lu, K Lee, Y Li, Q Zhai; (VI) Manuscript writing: All authors; (VII) Final approval of manuscript: All \\ authors. \\ "These authors contributed equally to this work. \\ Correspondence to: Shusen Wang, MD. Department of Medical Oncology, Sun Yat-sen University Cancer Center; State Key Laboratory of \\ Oncology in South China, Collaborative Innovation Center for Cancer Medicine, 651 Dongfeng Road East, Guangzhou 510060, China. \\ Email: wangshs@sysucc.org.cn.
}

Background: The role of local treatment for liver oligometastases in breast cancer patients has been controversial. The aim of the study was to evaluate the prognostic factors for overall survival (OS) and progression-free survival (PFS) after hepatic local treatment and to explore appropriate therapy strategy in breast cancer patients with liver oligometastases.

Methods: A cohort of 91 patients with oligometastatic liver lesions identified from around 34,000 breast cancer patients between 2002 and 2018 were retrospectively reviewed. We collected and analyzed their clinicopathologic and outcome data.

Results: With a median follow up of 20.6 months [standard deviations (SD): 35.4], median OS and median PFS were 75.1 months [95\% confidence interval (CI): $14.3-135.9$ months] and 7.4 months (95\% CI: 4.410.4 months), respectively. N3 stage, triple-negative breast cancer (TNBC) subtype, progressive disease (PD) after preoperative systemic therapy and older age of operation were associated with worse OS and PFS while postoperative systemic therapy alone, preoperative [partial response (PR)/stable disease (SD)] combined with postoperative systemic therapy indicated improved OS and PFS by univariate analysis. On multivariate analysis, N3 stage [hazard ratios (HR) 23.567; 95\% CI: 1.751-317.277; P=0.017] and TNBC subtype (HR 10.758; 95\% CI: 1.120-103.301; P=0.040) were related to worse OS. Likewise, N3 stage (HR 3.324; $95 \%$ CI 1.604-6.890; $\mathrm{P}=0.001$ ) and TNBC subtype (HR 3.134; 95\% CI: 1.015-9.674; $\mathrm{P}=0.047$ ) remained associated with worse PFS. The postoperative mortality and morbidity were $0 \%$ and $1.099 \%$, respectively.

Conclusions: In well-selected patients, local treatment of breast cancer liver oligometastases is safe and achieves relatively long OS, except patients with advanced $\mathrm{N}$ stage or aggressive subtype. Postoperative systemic treatment and effective preoperative systemic therapy combined with postoperative systemic therapy are the recommended treatment strategies.

Keywords: Breast cancer; oligometastasis; local therapy; prognostic factor; treatment strategy

Submitted Aug 29, 2019. Accepted for publication Dec 26, 2019.

doi: $10.21037 /$ tcr.2019.12.93

View this article at: http://dx.doi.org/10.21037/tcr.2019.12.93 


\section{Introduction}

Breast cancer is the most common malignant tumor in women. There were approximately 269,000 cases and about 70,000 deaths of breast cancer in China in 2015, accounting for $15 \%$ and $7 \%$ of female morbidity and mortality, respectively (1). Around $30 \%$ of breast cancer patients will eventually develop distant metastatic disease (2). Current guidelines suggest breast cancer patients with distant metastases receive palliative systemic treatment. Although systemic therapy for metastatic breast cancer has made great progress in recent years, metastasis is still related to poor prognosis.

The concept of an oligometastatic disease was first proposed by Hellman et al. $(3,4)$. In the 4th ESO-ESMO International Consensus Guidelines for Advanced Breast Cancer (ABC4), oligo-metastatic disease is defined as low volume metastatic disease with limited number and size of metastatic lesions (up to five and not necessarily in the same organ), potentially amenable for local treatment, aimed at achieving a complete remission status. Only $5-25 \%$ of distant metastatic breast cancer patients will develop isolated liver metastases and be eligible for liverdirected local treatment (5). For these patients, a recent meta-analysis reported a median 5-year survival of $39 \%$ following aggressive surgical resection of breast cancer liver metastases (BCLM) (6). The poor results achieved by systemic treatment alone and the potential benefit of local treatment have become the driving force behind the advocates for local treatment of oligo-metastatic lesions, especially when they respond well to systemic therapy. However, liver metastases of colorectal cancer are the most commonly resected liver metastases and the indication for BCLM is less well defined $(7,8)$. Current guidelines don't mention hepatic local treatment combined with systemic therapy as an option for liver oligometastases of breast cancer. In present, few studies have been done to compare the effect of local treatment and systemic therapy on liver oligometastases of breast cancer. The present study aims to identify possible prognostic factors of local treatment in breast cancer patients with liver oligometastases. Furthermore, we focused on breast cancer patients with liver oligometastases who underwent local treatment combined with systemic treatment to explore better treatment options for these patients.

\section{Methods}

\section{Patient population}

Data of around 34,000 patients with breast cancer at our institution between January 2002 and September 2018 were retrospectively reviewed after Institutional Review Board approval. Only breast cancer patients with histologically confirmed diagnosis of liver oligometastases undergoing hepatic local treatment were included. A total of 91 patients were finally enrolled in this study. For each patient, we collected age, gender and clinicopathologic data on the primary tumor including TNM stage, type of surgery, receptor status, and adjuvant/neoadjuvant treatment, as well as information on the BCLM, systemic therapy before/ after hepatic local treatment and response to preoperative systemic therapy.

\section{Statistical analysis}

Categorical variables were reported as whole numbers and percentages, while continuous variables were described as medians with standard deviations (SD). Overall survival (OS) was defined as the time from the date of hepatic local treatment to the date of death or date of last available follow-up. Progression-free survival (PFS) was the time from the date of hepatic local treatment to the date of progression or date of last available follow-up. The OS and PFS were estimated using the Kaplan-Meier method and compared using the log-rank test. To identify independent predictors of OS and PFS, factors with a univariate significance of $\mathrm{P}<0.10$ and potential clinical significance were entered into Cox proportional hazard models. Results from the Cox proportional hazards models were reported as hazard ratios (HR) with corresponding 95\% confidence intervals $(95 \% \mathrm{CI})$. All tests were two-tailed and statistical significance was set at $\mathrm{P} \leq 0.05$. All statistical analyses were performed with SPSS version 24.0 (IBM, New York, USA).

\section{Results}

\section{Demographic and clinicopatbologic features}

Between March 2002 and September 2018, a total of 91 breast cancer patients who underwent local therapy for BCLM were enrolled in this retrospective study. The 
clinicopathologic characteristics of all the patients are summarized in Table 1. Median patient age was 46.0 years old and 90 patients were female. Primary breast tumors of 42 patients were classified as either T1 or T2 $(n=42$, $75.0 \%)$ while $14(25.0 \%)$ patients had an advanced $\mathrm{T} 3$ or T4 primary breast cancer; $15.1 \%$ of patients $(n=11)$ had a primary breast tumor with lymph node stage of N3. The surgical approach of primary tumors used most was modified radical mastectomy $(\mathrm{n}=64,76.2 \%)$. Forty-eight $(65.8 \%)$ patients had a tumor with positive estrogen receptor status, while $48(67.6 \%)$ patients had a progesterone receptorpositive tumor. Twenty-five (38.5\%) patients had a HER2/ neu positive tumor and $5(7.7 \%)$ patients had a tumor of triple-negative breast cancer (TNBC) subtype. Primary tumor of $40(81.6 \%)$ patients is $\mathrm{Ki}-67>14 \%$. Most patients $(\mathrm{n}=60,67.4 \%)$ accepted ablation of liver oligometastases. As for the systemic treatment of BCLM, 57 patients received chemotherapy, 22 patients received hormonal therapy and 19 received biological therapy. Twenty-one (63.6\%) patients achieved partial response (PR)/stable disease (SD) among 39 patients who accepted preoperative systemic therapy.

The median disease-free survival (DFS) between primary tumor and BCLM were 21.5 months. The median number of BCLM was 1.0 while the median maximum tumor size was $2.6 \mathrm{~cm}$. The majority of BCLM located in unilateral lobe $(\mathrm{n}=66,76.2 \%)$. The median age of patients at local therapy of BCLM was 48 years old. At the time of local treatment of BCLM, 31 (73.8\%) patients had an estrogen receptor-positive tumor, while $21(50 \%)$ patients had a tumor with positive progesterone receptor status. After the operation of BCLM, 57 patients accepted systemic therapy.

\section{Long-term overall and PFS}

Median OS was 75.1 months (95\% CI: 14.3-135.9) (Figure 1A) with a median follow-up of 20.6 months (SD: 35.4). During the follow-up, a total of 23 (25.3\%) patients died. Sixteen $(17.6 \%)$ patients are alive more than 5 years since the operation of liver metastases with a longest survival of almost 16.6 years (198.6 months). Following local treatment of BCLM, recurrence occurred in 68 (79.1\%) patients with a median PFS of 7.4 months $(95 \%$ CI: 4.4-10.4) (Figure 1B).

Tables 2 and 3 showed the univariate and multivariate Cox regression analysis for predicting OS and PFS following local therapy of BCLM, respectively. On univariate analysis, $\mathrm{N} 3$ stage of primary tumor (HR 3.784; 95\% CI: 1.16-12.343; P=0.027), TNBC subtype (HR 8.514;
95\% CI: 2.158-33.600; $\mathrm{P}=0.002$ ), progressive disease (PD) after preoperative systemic therapy (HR 7.874; 95\% CI: 1.544-40.166; $\mathrm{P}=0.013$ ) and older age of operation (HR 1.039; $95 \%$ CI: $1.003-1.076 ; \mathrm{P}=0.031)$ were associated with worse OS while DFS of primary tumor surgery more than one year (HR 0.318; 95\% CI: 0.112-0.905; $\mathrm{P}=0.032$ ), postoperative systemic therapy alone (HR $0.152 ; 95 \% \mathrm{CI}$ : 0.034-0.679; $\mathrm{P}=0.014)$ and preoperative $(\mathrm{PR} / \mathrm{SD})$ combined with postoperative systemic therapy (HR $0.083 ; 95 \% \mathrm{CI}$ : 0.009-0.798; $\mathrm{P}=0.031$ ) indicated improved OS (Table 2). Meanwhile, N3 stage of primary tumor (HR 3.325; 95\% CI: 1.670-6.622; $\mathrm{P}=0.001$ ), TNBC subtype (HR 3.517; 95\% CI: $1.182-10.461 ; \mathrm{P}=0.024), \mathrm{PD}$ after preoperative systemic therapy (HR 2.421; 95\% CI: 1.029-5.694; $\mathrm{P}=0.043$ ), older age of operation (HR 1.033; 95\% CI: 1.012-1.054; P=0.002) were associated with worse PFS following local therapy of BCLM while systemic therapy (HR 0.359; 95\% CI: 0.1880.685; $\mathrm{P}=0.002$ ), postoperative systemic therapy alone (HR 0.256 ; $95 \%$ CI: $0.123-0.535 ; \mathrm{P}<0.001)$ and preoperative (PR/SD) combined with postoperative systemic therapy (HR 0.214, 95\% CI: 0.088-0.517, $\mathrm{P}=0.001$ ) indicated improved PFS (Table 3).

On multivariate analysis, N3 stage of primary tumor (HR 23.567; 95\% CI: 1.751-317.277; $\mathrm{P}=0.017$ ) and TNBC subtype (HR 10.758; 95\% CI: 1.120-103.301; $\mathrm{P}=0.040$ ) were found to be significant predictors of worse OS. Likewise, N3 stage of primary tumor (HR 3.324; 95\% CI: 1.604-6.890; $\mathrm{P}=0.001$ ) and TNBC subtype (HR 3.134; 95\% CI: 1.015-9.674; $\mathrm{P}=0.047)$ remained associated with worse PFS following operation of BCLM.

\section{Short-term outcome}

As for the safety, there were no related deaths of local treatment and only one patient (1.099\%) experienced a complication of needle-track bleeding among all series.

\section{Discussion}

Although metastatic breast cancer is a systemic disease, the effects of systemic treatment are not satisfactory. Despite the rapid development of systemic therapy for breast cancer patients over the past few decades, the 5-year survival rate of BCLM patients remains low, which is only $4-12 \%$ (9-11). According to the previous findings, the 5-year survival rate of patients receiving local treatment of BCLM is $27-50 \%$ (12). A systemic review analyzed 19 studies with 553 cases of BCLM and reported the median OS of 
Table 1 Clinicopathologic characteristics of primary breast cancer and breast cancer liver metastases

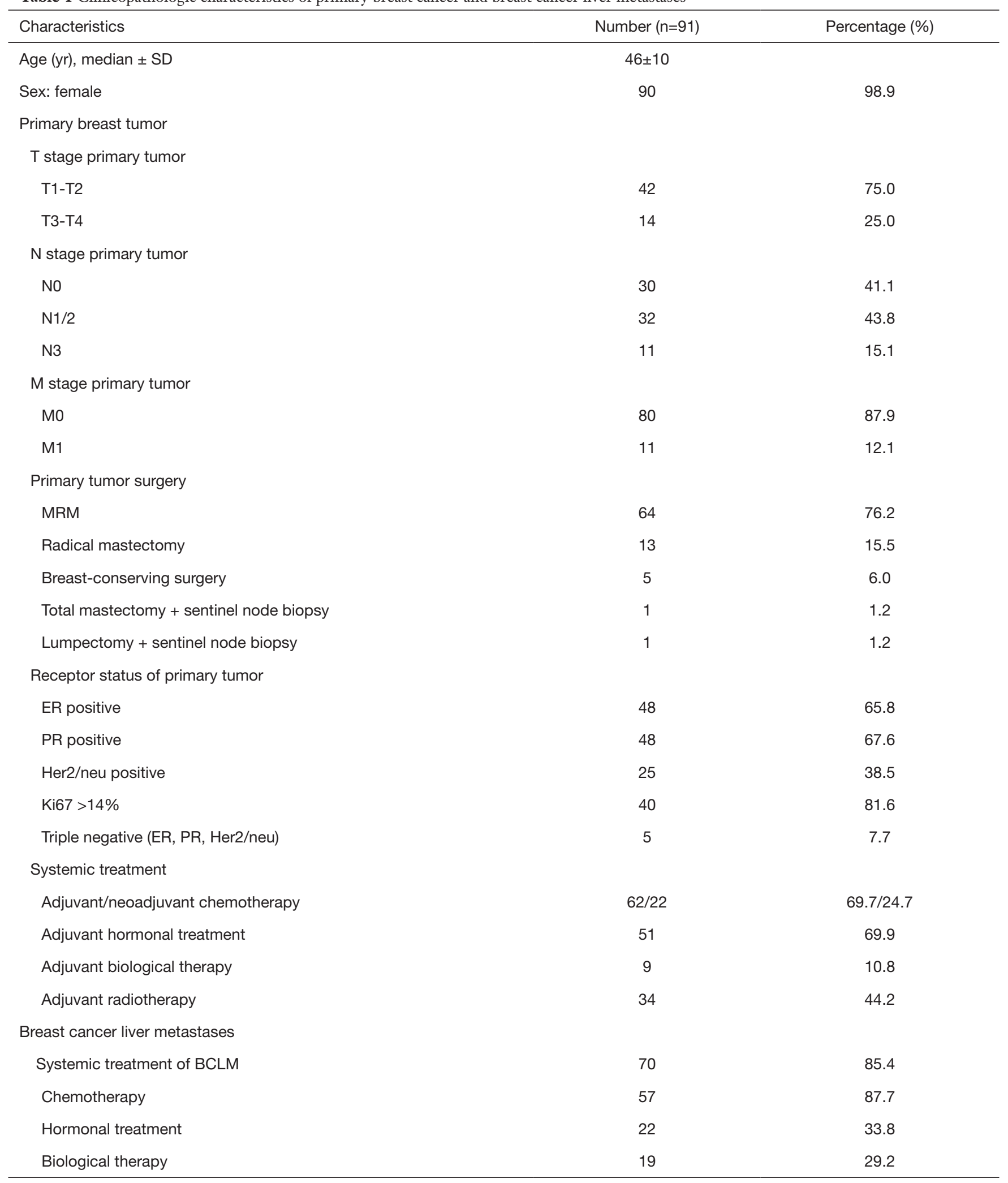

Table 1 (continued) 
Table 1 (continued)

\begin{tabular}{|c|c|c|}
\hline Characteristics & Number $(\mathrm{n}=91)$ & Percentage (\%) \\
\hline Response to preoperative systemic therapy (PR+SD/PD) & $21 / 12$ & $63.6 / 36.4$ \\
\hline Median interval months between primary and $B C L M \pm S D$ & $21.5 \pm 52.1$ & \\
\hline Median number of $B C L M \pm S D$ & $1.0 \pm 0.7$ & \\
\hline \multicolumn{3}{|l|}{ Distribution } \\
\hline Unilateral & 66 & 76.7 \\
\hline Bilateral & 20 & 23.3 \\
\hline Median age at local treatment of BCLM $\pm S D(y r)$ & $48 \pm 11$ & \\
\hline \multicolumn{3}{|l|}{ Type of local treatment } \\
\hline Surgery & 29 & 32.6 \\
\hline \multicolumn{3}{|l|}{ Receptor status of BCLM } \\
\hline ER positive/ER negative & $31 / 11$ & $73.8 / 26.2$ \\
\hline PR positive/PR negative & $21 / 21$ & $50.0 / 50.0$ \\
\hline HER2 positive/HER2 negative & $11 / 27$ & 28.9/71.1 \\
\hline Post operation systemic therapy & 57 & 71.2 \\
\hline
\end{tabular}

BCLM, breast cancer liver metastases; PR, partial response; SD, stable disease; PD, progressive disease.
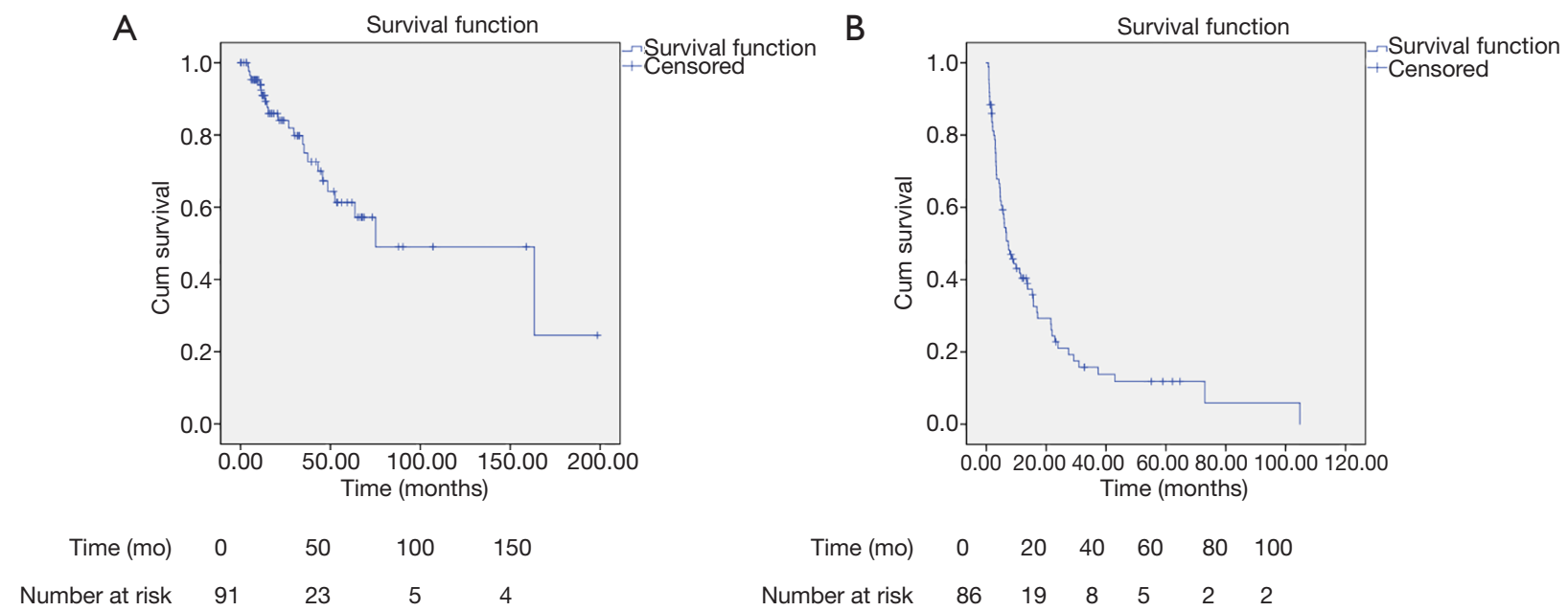

Number at risk $\quad 91 \quad 23 \quad 5 \quad 4$

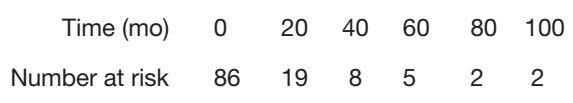

Figure 1 Kaplan-Meier estimates for (A) overall survival and (B) progression-free survival. 
Table 2 Univariate and multivariate analysis of overall survival after local treatment of BCLM

\begin{tabular}{|c|c|c|c|c|c|c|}
\hline Variables & \multicolumn{3}{|c|}{ Univariable } & \multicolumn{3}{|c|}{ Multivariable } \\
\hline \multicolumn{7}{|l|}{ T stage primary tumor } \\
\hline T1-T2 & Ref & - & - & & & \\
\hline T3-T4 & 0.249 & $0.031-1.983$ & 0.189 & & & \\
\hline N0-N2 & Ref & - & - & & & \\
\hline N3 & 3.784 & $1.16-12.343$ & $0.027^{\star}$ & 23.567 & $1.751-317.277$ & $0.017^{*}$ \\
\hline \multicolumn{7}{|l|}{ Breast cancer subtypes of primary tumor } \\
\hline TNBC & 8.514 & $2.158-33.600$ & $0.002^{*}$ & 10.758 & $1.120-103.301$ & $0.040^{*}$ \\
\hline \multicolumn{7}{|l|}{ Response to preoperative systemic therapy } \\
\hline$P R+S D$ & Ref & - & - & & & \\
\hline PD & 7.874 & $1.544-40.166$ & $0.013^{*}$ & & & \\
\hline Systemic therapy & 0.330 & $0.104-1.046$ & 0.060 & 709255.538 & & 0.977 \\
\hline \multicolumn{7}{|l|}{ Treatment strategy } \\
\hline No systemic therapy & Ref & - & - & & & \\
\hline Preoperative systemic therapy alone (PR/SD) & 0.775 & $0.138-4.341$ & 0.771 & & & \\
\hline Preoperative systemic therapy alone (PD) & 1.136 & $0.197-6.548$ & 0.887 & & & \\
\hline Age of operation & 1.039 & $1.003-1.076$ & $0.031^{*}$ & & & \\
\hline \multicolumn{7}{|l|}{ DFS of primary tumor surgery } \\
\hline$\leq 1$ year & Ref & - & - & & & \\
\hline$>1$ year & 0.318 & $0.112-0.905$ & $0.032^{*}$ & 0.543 & $0.068-4.306$ & 0.563 \\
\hline Diameter of BCLM & 1.027 & $0.751-1.406$ & 0.866 & & & \\
\hline
\end{tabular}

* $\mathrm{P}<0.05$ denotes statistical significance. BCLM, breast cancer liver metastases; PR, partial response; SD, stable disease; PD, progressive disease; TNBC, triple-negative breast cancer.

patients receiving local treatment of liver metastases was 40 months (15-74 months) with the median 5-year survival rate of $40 \%(21-80 \%)$ (13). Charalampoudis et al. found that the median OS of BCLM patients undergoing surgery for liver metastases was 41 months, with a median PFS of 11.5 months in a recent meta-analysis (6). The results of a multicenter retrospective study showed the median survival time of 67 BCLM patients who underwent hepatectomy was 57.59 months and suggested that an interval of more than 2 years between breast cancer surgery and liver metastasis might be an indication of liver surgery in BCLM patients (14). A case-control study by Sadot et al. focused on 
Table 3 Univariate and multivariate analysis of progression-free survival after local treatment of BCLM

\begin{tabular}{|c|c|c|c|c|c|c|}
\hline Variables & \multicolumn{3}{|c|}{ Univariable } & \multicolumn{3}{|c|}{ Multivariable } \\
\hline \multicolumn{7}{|l|}{ T stage primary tumor } \\
\hline $\mathrm{T} 1-\mathrm{T} 2$ & Ref & - & - & & & \\
\hline T3-T4 & 0.621 & $0.297-1.298$ & 0.205 & & & \\
\hline N0-N2 & Ref & - & - & & & \\
\hline N3 & 3.325 & $1.670-6.622$ & $0.001^{*}$ & 3.324 & $1.604-6.890$ & $0.001^{*}$ \\
\hline \multicolumn{7}{|l|}{ Breast cancer subtypes of primary tumor } \\
\hline TNBC & 3.517 & $1.182-10.461$ & $0.024^{*}$ & 3.134 & $1.015-9.674$ & $0.047^{*}$ \\
\hline \multicolumn{7}{|l|}{ Response to preoperative systemic therapy } \\
\hline $\mathrm{PR}+\mathrm{SD}$ & Ref & - & - & & & \\
\hline PD & 2.421 & $1.029-5.694$ & $0.043^{*}$ & & & \\
\hline Systemic therapy & 0.359 & $0.188-0.685$ & $0.002^{*}$ & 0.477 & $0.215-1.055$ & 0.067 \\
\hline \multicolumn{7}{|l|}{ Treatment strategy } \\
\hline No systemic therapy & Ref & - & - & & & \\
\hline Preoperative systemic therapy alone (PR/SD) & 1.146 & $0.396-3.313$ & 0.802 & & & \\
\hline Preoperative systemic therapy alone (PD) & 1.309 & $0.415-4.130$ & 0.646 & & & \\
\hline Age of operation & 1.033 & $1.012-1.054$ & $0.002^{*}$ & & & \\
\hline \multicolumn{7}{|l|}{ DFS of primary tumor surgery } \\
\hline$\leq 1$ year & Ref & - & - & & & \\
\hline$>1$ year & 1.004 & $0.474-2.127$ & 0.992 & & & \\
\hline Diameter of BCLM & 1.100 & $0.983-1.231$ & 0.097 & & & \\
\hline
\end{tabular}

${ }^{*}, \mathrm{P}<0.05$ denotes statistical significance. BCLM, breast cancer liver metastases; PR, partial response; SD, stable disease; PD, progressive disease; TNBC, triple-negative breast cancer.

the benefits of surgery for isolated BCLM (15). This study has shown no significant difference in OS between the surgical and medical cohorts (median OS: 50 vs. 45 months; 5-year OS: 38\% vs. 39\%). However, 69 patients undergoing surgical treatment had a median recurrence-free interval (RFI) of 28.5 months. Therefore, the authors concluded that although hepatic local treatment was not associated with survival advantages, longer RFI achieved by surgery could potentially provide patients with a period of time during which they might avoid systemic therapy. Another recent study by Spolverato et al. constructed a decisionanalytic Markov model and found that liver resection of 
BCLM was cost-effective compared to systemic therapy alone, especially in estrogen receptor-positive tumors or when using modern drugs (16). The evidence to support stereotactic ablative radiotherapy (SABR) as an effective treatment for oligometastatic disease mainly comprises of retrospective series and single-arm prospective trials. There were increasing prospective randomized controlled trials to evaluate the efficacy and safety of SABR as oligometastasisdirected treatment (17). From the multi-national SABRCOMET randomized phase II screening trial of 99 oligometastatic patients ( $\mathrm{n}=18$ with breast cancer), the use of SABR versus standard of care therapy alone, significantly increased the median PFS (12 vs. 6 months) and the median OS difference (41 vs. 28 months) (18). Besides, the use of SABR was not associated with a quality of life detriment (19). Another prospective clinical trial indicated that in selected breast cancer patients with bone-only oligometastatic disease, SABR could be a feasible, well-tolerated and effective treatment option (20). Long-term results of a recent phase II study suggest the efficacy and safety of SBRT for unresectable liver metastases and univariate analysis showed that favorable primary site (breast, colorectal and gynecological) of metastases improved survival (21). In conclusion, current studies suggested that local treatment of BCLM in carefully selected patients should be considered.

Our study showed a median OS of 75.1 months and the median PFS was 7.4 months. The difference could be due to the following reasons. The first and foremost, the fact that the participants were all oligometastatic breast cancer patients with relatively low tumor load might explain the relatively longer OS. Besides, the repetition of local treatment and subsequent systemic therapy could also lead to survival benefit. In addition, PFS in our study refers to the period between the date of the first hepatic operation and the date of progression or last follow-up while other studies might set it from the time of the last hepatic operation. We set PFS in this way to avoid the influence of systemic therapy because patients would receive systemic treatment between the intervals of hepatic operations. Approximately $9 \%$ of patients received more than one local treatment in the present study. Furthermore, tumors of a significant proportion of patients $(19,8$ and 2 patients, respectively) had not progressed during the follow-up of 20, 40 and 100 months, as shown in Figure $1 B$. It suggested that the median PFS might not reflect the full picture of the PFS in our study.

According to the historical published studies, prognostic factors related to surgical outcome of liver metastases of breast cancer were axillary lymph node status, hormone receptor status, surgical margin, number of liver metastases, and disease-free interval (DFI) (22-25). The purpose of this study is to provide some suggestions for how to select appropriate breast cancer patients of liver oligometastases for local treatment. We found that patients whose primary breast cancer with lymph node stage of $\mathrm{N} 3$ and TNBC subtypes have worse OS and PFS after operation of liver lesions. It's suggested that oligo-metastatic patients with advanced $\mathrm{N}$ stage or aggressive subtype of the primary breast tumor might not be recommended to receive local treatment of liver lesions.

At present, there are many unresolved questions related to local treatment in breast cancer patients with liver oligometastases. Which therapy strategy is more beneficial among local treatment alone, systemic treatment alone or systemic treatment combined with local treatment? Is there any difference between systemic therapy before or after local treatment? Does the response to systemic therapy before local treatment affect the prognosis? Our study collected data of breast cancer patients with liver oligometastases trying to answer the above questions. On univariate analysis, we found that patients receiving local treatment combined with systemic treatment achieved significantly longer PFS than those undergoing local treatment alone while there was a nearly significant difference $(\mathrm{P}=0.060)$ in OS. In addition, we compared the clinical outcome of patients who accepted different treatment strategies. As a result, OS and PFS of patients underwent post-operational systemic therapy alone were significantly better than those received local therapy alone. We further compared the outcome of patients with different response to systemic therapy before hepatic treatment and revealed that the OS and PFS of the $\mathrm{PR} / \mathrm{SD}$ group were significantly improved than the PD group. Besides, if patients with good response (PR or SD) to preoperative systemic therapy also received postoperative systemic therapy, the OS and PFS were significantly improved than the local therapy alone group. By contrast, if patients with poor response (PD) to preoperative systemic therapy accepted postoperative systemic therapy, the OS and PFS were not significantly different from the local therapy alone group. Furthermore, despite of different responses, the two preoperative systemic therapy alone groups both have no OS and PFS advantages. Unexpectedly, the presence of systemic therapy with local treatment was not an independent predictor of prognosis on multivariate analysis, probably on account of the small sample size of 
our study. Studies with large sample exploring these issues might get more satisfactory results. Nevertheless, we inferred from our results that breast cancer patients with liver oligometastases should consider two types of treatment strategies. One is to receive effective preoperative systemic therapy combined with postoperative systemic treatment and the other is to accept postoperative systemic therapy. These results also remind us to avoid the absence of systemic therapy after local treatment when treating these oligo-metastatic patients in the clinical practice.

The current study is a retrospective, single-center, smallsample study with highly selected patients. However, our results should not be dismissed as selection bias since they are rarely achieved by conventional systemic treatment. We not only investigated the prognostic factors of local treatment for oligometastases in breast cancer patients but also innovatively explored the appropriate treatment strategy for them. Further study in a larger population and randomized controlled trials are necessary to validate our results and find a way out of current dilemma of treatment for oligometastases in breast cancer patients. Furthermore, constructing a prognostic model and performing genetic testing on patients of oligometastatic breast cancer might achieve precise and individualized treatment in the future.

In conclusion, local treatment of breast cancer liver oligometastases is safe and achieves relatively long OS in well-selected patients. However, patients with advanced $\mathrm{N}$ stage or aggressive subtype of the primary tumor might not be appropriate to receive local treatment of liver lesions. Postoperative systemic treatment and effective preoperative systemic therapy combined with postoperative systemic therapy are the recommended treatment strategies.

\section{Acknowledgments}

We thank the patients who participated in this study. We thank all authors for their cooperation.

Funding: None.

\section{Footnote}

Conflicts of Interest: All authors have completed the ICMJE uniform disclosure form (available at http://dx.doi. org/10.21037/tcr.2019.12.93). The authors have no conflicts of interest to declare.

Ethical Statement: The authors are accountable for all aspects of the work in ensuring that questions related to the accuracy or integrity of any part of the work are appropriately investigated and resolved. This study was approved by the committee of Institutional Review Board of Sun Yat-Sen University Cancer Center (B2019-197-01). All patients at our institution are systemically asked to consent to the use of anonymous data for analysis and publication. No identifiable data was collected. All procedures performed in studies involving human participants were in accordance with the ethical standards of the institutional and/or national research committee and with the 1964 Helsinki declaration and its later amendments or comparable ethical standards.

Open Access Statement: This is an Open Access article distributed in accordance with the Creative Commons Attribution-NonCommercial-NoDerivs 4.0 International License (CC BY-NC-ND 4.0), which permits the noncommercial replication and distribution of the article with the strict proviso that no changes or edits are made and the original work is properly cited (including links to both the formal publication through the relevant DOI and the license). See: https://creativecommons.org/licenses/by-nc-nd/4.0/.

\section{References}

1. Chen $\mathrm{W}$, Zheng R, Baade PD, et al. Cancer statistics in China, 2015. CA Cancer J Clin 2016;66:115-32.

2. Huober J, Thürlimann B. The Role of Combination Chemotherapy in the Treatment of Patients with Metastatic Breast Cancer. Breast Care (Basel) 2009;4:367-72.

3. Hellman S, Weichselbaum RR. Oligometastases. J Clin Oncol 1995;13:8-10.

4. Weichselbaum RR, Hellman S. Oligometastases revisited. Nat Rev Clin Oncol 2011;8:378-82.

5. Insa A, Lluch A, Prosper F, et al. Prognostic factors predicting survival from first recurrence in patients with metastatic breast cancer: analysis of 439 patients. Breast Cancer Res Treat 1999;56:67-78.

6. Charalampoudis P, Mantas D, Sotiropoulos GC, et al. Surgery for liver metastases from breast cancer. Future Oncol 2015;11:1519-30.

7. Tomlinson JS, Jarnagin WR, DeMatteo RP, et al. Actual 10-year survival after resection of colorectal liver metastases defines cure. J Clin Oncol 2007;25:4575-80.

8. Huang F, Wu G, Yang K. Oligometastasis and oligorecurrence: more than a mirage. Radiat Oncol 2014;9:230-8. 
9. Ge QD, Lv N, Kong YN, et al. Clinical characteristics and survival analysis of breast cancer molecular subtypes with hepatic metastases. Asian Pac J Cancer Prev 2012;13:5081-6.

10. Lobbezoo DJ, van Kampen RJ, Voogd AC, et al. Prognosis of metastatic breast cancer subtypes: the hormone receptor/HER2-positive subtype is associated with the most favorable outcome. Breast Cancer Res Treat 2013;141:507-14.

11. O'Reilly SM, Richards MA, Rubens RD. Liver metastases from breast cancer: The relationship between clinical, biochemical and pathological features and survival. Eur J Cancer 1990;26:574-7.

12. Ruiz A, Sebagh M, Wicherts DA, et al. Long-term survival and cure model following liver resection for breast cancer metastases. Breast Cancer Res Treat 2018;170:89-100.

13. Chua TC, Saxena A, Liauw W, et al. Hepatic resection for metastatic breast cancer: a systematic review. Eur J Cancer 2011;47:2282-90.

14. He X, Zhang Q, Feng Y, et al. Resection of liver metastases from breast cancer: a multicentre analysis. Clin Transl Oncol 2019. [Epub ahead of print].

15. Sadot E, Lee SY, Sofocleous CT, et al. Hepatic Resection or Ablation for Isolated Breast Cancer Liver Metastasis: A Case-control Study With Comparison to Medically Treated Patients. Ann Surg 2016;264:147-54.

16. Spolverato G, Vitale A, Bagante F, et al. Liver Resection for Breast Cancer Liver Metastases: A Cost-utility Analysis. Ann Surg 2017;265:792-9.

17. Al-Shafa F, Arifin AJ, Rodrigues GB, et al. A Review of Ongoing Trials of Stereotactic Ablative Radiotherapy for Oligometastatic Cancers: Where Will the Evidence Lead? Front Oncol 2019;9:543-8.

Cite this article as: Jiang $\mathrm{K}$, Xia W, Hong $\mathrm{R}, \mathrm{Xu} F$, Zheng Q, Lu Q, Lee K, Li Y, Zhai Q, Shi Y, Yuan Z, Wang S. Local treatment for liver oligometastases in breast cancer patients: identification of prognostic factors and exploration of appropriate treatment strategy. Transl Cancer Res 2020;9(2):1225-1234. doi: 10.21037/tcr.2019.12.93
18. Palma DA, Olson R, Harrow S, et al. Stereotactic ablative radiotherapy versus standard of care palliative treatment in patients with oligometastatic cancers (SABRCOMET): a randomised, phase 2, open-label trial. Lancet 2019;393:2051-8.

19. Olson R, Senan S, Harrow S, et al. Quality of Life Outcomes After Stereotactic Ablative Radiation Therapy (SABR) Versus Standard of Care Treatments in the Oligometastatic Setting: A Secondary Analysis of the SABR-COMET Randomized Trial. Int J Radiat Oncol Biol Phys 2019;105:943-7.

20. David S, Tan J, Savas P, et al. Stereotactic ablative body radiotherapy (SABR) for bone only oligometastatic breast cancer: A prospective clinical trial. Breast 2020;49:55-62.

21. Scorsetti M, Comito T, Clerici E, et al. Phase II trial on SBRT for unresectable liver metastases: long-term outcome and prognostic factors of survival after 5 years of follow-up. Radiat Oncol 2018;13:234-43.

22. Margonis GA, Buettner S, Sasaki K, et al. The role of liver-directed surgery in patients with hepatic metastasis from primary breast cancer: a multi-institutional analysis. HPB (Oxford) 2016;18:700-5.

23. Zegarac M, Nikolic S, Gavrilovic D, et al. Prognostic factors for longer disease free survival and overall survival after surgical resection of isolated liver metastasis from breast cancer. J BUON 2013;18:859-65.

24. Treska V, Cerna M, Kydlicek T, et al. Prognostic factors of breast cancer liver metastasis surgery. Arch Med Sci 2015;11:683-5.

25. Hoffmann K, Franz C, Hinz U, et al. Liver resection for multimodal treatment of breast cancer metastases: identification of prognostic factors. Ann Surg Oncol 2010;17:1546-54. 